\title{
ENVIRONMENTAL AND SOCIAL FACTORS MOTIVATE THE EMERGENCE OF SYSTEMATIC CATEGORIES AND SIGNS
}

\author{
Jonas Nölle ${ }^{* 1,3}$, Marlene Staib ${ }^{2}$, Riccardo Fusaroli ${ }^{2,3}$, and Kristian Tylén ${ }^{2}$ \\ *Corresponding Author: j.nolle@sms.ed.ac.uk \\ ${ }^{1}$ Centre for Language Evolution, University of Edinburgh, UK \\ ${ }^{2}$ Center for Semiotics, Aarhus University, Denmark \\ ${ }^{3}$ The Interacting Minds Centre, Aarhus University, Denmark
}

While arbitrariness has long been considered a hallmark of human language, there have been increasing discussions about non-arbitrary relationships between form and meaning such as iconicity and systematicity (Dingemanse et al., 2015). We argue that these phenomena are not just two facets of non-arbitrariness, but serve orthogonal functions in the scaffolding of an efficient communication system. Iconicity is usually associated with learning and bootstrapping (Imai \& Kita, 2014), while systematicity has been shown to emerge in the lab when iconicity is inhibited (Roberts et al., 2015; Verhoef et al., 2016) or when participants were provided with pre-established combinatorial categories (Theisen et al., 2010).

Building on this work, we tested in a series of dyadic silent gesture experiments whether systematicity is functionally adaptive and could also emerge in competition to iconicity in response to particular environmental and social factors. We hypothesized that structure and openness of the environment as well as working memory constrains (Christiansen \& Chater, 2016) could affect the degree of systematicity as well as which referential features become systematized. In two experiments, participants had to communicate stimuli depicting stylized characters that afforded both iconic and systematic gestures (see Fig. 1A). Experiment 1 tested a) whether functional adaptivity of a given trait, based on its distribution in the referential environment (consisting of 14 stimuli drawn from a set of 24) and thus its discriminative value, would affect the likelihood of this trait being systematized and $b$ ) the impact of an expanding referential environment. In a $2 \times 2$ design we varied the distribution of PET vs. GENDER (7:7 vs. 10:4) and the openness of the environment (an expanding meaning space in the open vs. constant meanings across trials in the closed condition). Logistic mixed regression models (see supplementary materials) indicated that functionally more adaptive traits were indeed more likely to be systematized $(p<.01)$ while openness of the environment had no significant effect (see Fig. 1B). Debriefing revealed that participants in the open condition frequently realized that the changing competitor images never had to be signed, which narrowed down the search space rather than enlarging it. 


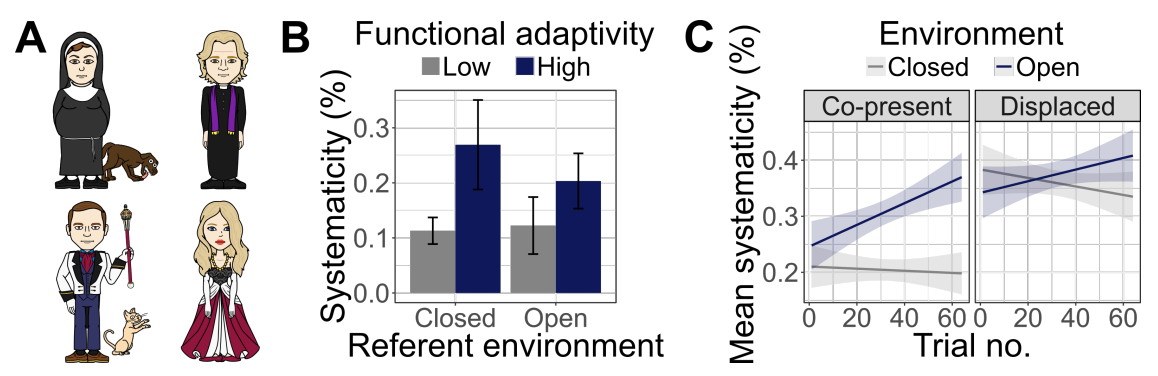

Figure 1. A Stimuli possessed various salient idiosyncratic features affording iconic descriptions (e.g., hat, scarf) as well as categorical traits (e.g., FEMALE, CLERICAL, PET) that could be fully systematized. B Systematicity by functional adaptivity and referent environment (exp. 1) C Mean proportion of systematic gestures by trial number (exp. 2). See supplementary materials for statistics.

Experiment 2 was designed to further explore the roles of informational bottlenecks. We improved the "openness" manipulation by increasing the number of stimuli (n=32) and referents per trial $(n=16)$ and randomly sampling both targets and competitors for each trial in the open condition, while the set remained the same across trials in the closed condition. In addition, we added "displacement" as a second factor. As in experiment 1, in the co-present condition, participant pairs could gesture while seeing the stimuli, whereas dyads in the displaced condition had to wait 3 seconds after the stimuli disappeared before they could gesture-effectively displacing the communicative from the referential context and simulating communication about absent entities. Our results indicate that displacement increased the tendency to systematize overall, while openness of the environment affected the temporal development of systematicity (see Fig. 1C). In the open condition systematicity kept increasing until the end of the experiment, while there was a decline in the closed condition that resembled the slope of both the closed and "pseudo"-open condition in experiment 1 , suggesting that a true open environment can override the tendency to fall back on simple iconic signs as communication becomes more efficient over time (Kirby et al., 2015).

Taken together, our results indicate that, given certain environmental affordances, systematicity can emerge in a novel communication system at the interaction level even in the presence of competing iconic solutions. In our experiment both systematic categories and signs emerged in response to their functional adaptivity to the environment and working memory constraints of the communicative situation (openness of the referential context, displacement). This could lead to variation that is amplified over cultural transmission (Kirby et al., 2015). Lastly, our study provides the first experimental semiotics study investigating the effect of displacement, a core property of language (Hockett, 1960) that has curiously been neglected in experimental studies so far. 


\section{Acknowledgements}

We would like to thank the Cognition and Behavior Lab at Aarhus BSS for facilities and equipment as well as Sergio Gonzales De La Higuera Rojo and Caroline Kildahl for coding of the video material. This work was funded by a Seed Grant of the Interactive Minds Centre as well as a funding from the School of Communication and Culture, Aarhus University.

\section{References}

Christiansen, M. H., \& Chater, N. (2016). The Now-or-Never bottleneck: A fundamental constraint on language. Behavioral and Brain Sciences, 39.

Dingemanse, M., Blasi, D. E., Lupyan, G., Christiansen, M. H., \& Monaghan, P. (2015). Arbitrariness, Iconicity, and Systematicity in Language. Trends in Cognitive Sciences, 19(10), 603-615.

Hockett, C. F. (1960). The Origin of Speech. Scientific American, 203(3), 88-97.

Imai, M., \& Kita, S. (2014). The sound symbolism bootstrapping hypothesis for language acquisition and language evolution. Phil. Trans. R. Soc. B, 369(1651), 20130298.

Kirby, S., Tamariz, M., Cornish, H., \& Smith, K. (2015). Compression and communication in the cultural evolution of linguistic structure. Cognition, 141, 87-102.

Roberts, G., Lewandowski, J., \& Galantucci, B. (2015). How communication changes when we cannot mime the world: Experimental evidence for the effect of iconicity on combinatoriality. Cognition, 141, 52-66.

Theisen, C. A., Oberlander, J., \& Kirby, S. (2010). Systematicity and arbitrariness in novel communication systems. Interaction Studies, 11(1), 14-32.

Verhoef, T., Kirby, S., \& de Boer, B. (2016). Iconicity and the Emergence of Combinatorial Structure in Language. Cognitive Science, 40(8), 1969-1994. 